\title{
Systematic study of inclusive hadron production spectra in collider experiments
}

\author{
A.Bylinkin, A.Rostovtsev \\ Institute for Theoretical and Experimental Physics, B.Cheremushkinskaya 25, 117218 Moscow, \\ Russia
}

DOI: http://dx.doi.org/10.5689/UA-PROC-2010-09/14

\begin{abstract}
A shape of invariant differential cross section for hadron production as function of transverse momentum is analysed. The systematic analysis of the available data demonstrates a need for a modification of the parameterization traditionally used to approximate the measured spectra. The properties of the new proposed parameterization are discussed.
\end{abstract}

\section{Introduction}

There exists a large body of experimental data on hadron production in high energy protonproton, photon-proton, photon-photon and heavy ion collisions. The spectra of hadrons produced in these collisions are characterized by an exponential behavior as function of transverse energy $\left(E_{T}\right)$ for the bulk of produced hadrons, which populate the low $E_{T}$ part of the spectra. This behavior resembles the Boltzmann-like spectrum in classical thermodynamics. The exponential shape of the spectra changes to a power law for high $E_{T}$ hadrons. This change is traditionally interpreted as an onset of the perturbative QCD regime of hadron production. These features of the spectra shape are found to be universal for any type of colliding particles. Therefore, it is tempting to find one universal smooth functional form, which describes the spectra of produced hadrons in the whole available $E_{T}$ range for different energies and types of colliding particles. The parameters of such universal functional form are expected to vary for different collision energies, types of colliding particles and types of produced hadrons. A study of the variations of these parameters provides a unique information on the hadron production dynamics.

In the present paper the experimentally measured inclusive spectra of long-lived charged particles (mainly charged pions) produced at central rapidities in the colliding particles center of mass system are analyzed. Further on it is assumed all charged particles being pions for simplicity. The present analysis is based on the published hadron production measurements made with $p p-$ collisions at ISR [1] and LHC [15], $p \bar{p}$-collisions at $S p \bar{p} S[2,3,4]$ and Tevatron [5, 6], $A u A u$-collisions with different centralities at RHIC [14], real $\gamma \gamma$-collisions at LEP [9, 11] and $\gamma p$-interactions with different values of photon virtuality $\left(Q^{2}\right)$ at HERA. The HERA data additionally allow to consider two different regimes of $\gamma p$-interactions: photoproduction at low values of $Q^{2}[7,8,10]$ and Deep Inelastic Scattering (DIS) for high $Q^{2}$ values $[12,13]$. The data for all these inclusive differential cross section measurements have been taken with a minimum bias trigger conditions and at center of mass energy $(\sqrt{s})$ ranging from 23 to $2360 \mathrm{GeV}$. 


\section{Modification of the Tsallis function}

A typical charged particle spectrum as function of transverse energy is traditionally fitted using the Tsallis-type function [16]

$$
\frac{d \sigma}{P_{T} d P_{T}}=\frac{A}{\left(1+\frac{E_{T}}{T \cdot n}\right)^{n}},
$$

where $P_{T}$ is transverse momentum of the produced particle, $E_{T}=\sqrt{P_{T}^{2}+M^{2}}$ with $\mathrm{M}$ equal to the pion mass. The parameterization (1) has only three free parameters: $A, T$ and $n$. While $A$ is an overall normalization, the $T$ and $n$ carry important information on the hadron production dynamics. Since for low $E_{T}$ values the parameterization (1) is reduced to the Boltzmann exponent $\sim \exp \left(-E_{T} / T\right)$, the parameter $T$ is a QCD analogy to a temperature in classical thermodynamics. Note, this analogy isn't straight forward, however.

In perturbative QCD calculations the value of the parameter $n$ depends on the shape of structure functions and partonic content of the colliding particles as well as on the differential cross sections of parton-parton interactions. The economic Tsallis-type parameterization (1) provides a good overall description of the spectrum, thus making the Tsallis-type function broadly used to fit the recent measurements at RHIC and LHC.

However, a closer look at the fits to the available data discloses systematic defects in these fits. In order to see these defects it is convenient to plot a ratio of the data to the fit function. Two such ratios are shown in Figure 1(a,b) for the data sets provided by the UA1 experiment with $p \bar{p}$ collisions at $\sqrt{s}=630 \mathrm{GeV}$ [4] (Fig 1a) and by RHIC $A u-A u$ collisions at $\sqrt{s}=$ $200 \mathrm{GeV} / \mathrm{N}[14]$ (Figure 1b). On both plots one observes broad dips and bumps around or above $1 \mathrm{GeV}$ scale.

The observed difference between the shapes of data spectra and function (1) is also typical for other available data sets, not shown here. This observation indicates that the true shape of the measured spectra doesn't follow exactly the Tsallis-type parameterization (1). Moreover, a larger mismatch between the shapes of the data spectrum and fit function (1) is found for $J / \Psi$ production at the Tevatron [17]. In the following an attempt is made to find a function which approximates the shape of the inclusive hadron production spectra better than the Tsallis parameterization.

To construct a new fit function a combination of two functional forms has been used: the exponential and power-law. The both functional forms must be the functions of scalars $P_{T}^{2}$ or $E_{T}^{k i n}=E_{T}-M$. This choice of these variables is very convenient since the both $P_{T}^{2}$ and $E_{T}^{\text {kin }}$ vary from zero to the corresponding kinematical limits. Non-true scalars like $P_{T}$ or $P_{T}^{3}$ have not been considered here. Finally, the parameterization

$$
\frac{d \sigma}{P_{T} d P_{T}}=A_{e} \exp \left(-E_{T}^{k i n} / T_{e}\right)+\frac{A}{\left(1+\frac{P_{T}^{2}}{T^{2} \cdot n}\right)^{n}},
$$

turned out to be in a good agreement with the data. The new ratios between data and fit function (2) are shown in Figure 1(c,d) for the considered above UA1 and RHIC data sets correspondingly. Figure $1(\mathrm{c}, \mathrm{d})$ demonstrates a significant improvement of the quality of the spectra shape approximation by function (2) with respect to that using the Tsallis-type function (1). In the following we present the arguments why this improvement is not a trivial result of increasing the number of free parameters in the fit function.

The most surprising feature of the new parameterization (2) is a strong correlation between the parameters $T_{e}$ and $T$. This correlation provides an additional constraint for the parame- 
terization (2) and therefore reduces a number of free parameters used in the fit. Though the physical origin of the observed correlation is not quite clear, the new constraint helps to minimize uncertainties of the parameter values obtained from the fits. This constraint will be used further on through this report.

The form of the parameterization (2) has a simple toy-model interpretation. Within this toy-model a small fraction of hadrons are produced directly in parton-parton interactions of the colliding particles. As required by the perturbative QCD the spectrum of particles produced in parton-parton interactions is described by a power law distribution. The rest bulk of irradiated hadrons represents a quasi-thermolized hadronic gas produced with a characteristic temperature $T_{e}$. The spectrum of hadrons in this gas has the Boltzman exponential shape. Interestingly, a sililar combination of the Boltzman-like and power law terms is observed in the photon energy spectra from the sun flares [18]. This illustrates a synergism of the thermal and non-thermal particles produced both in sun flares and in hadron interactions.

The relative contribution of the exponential and power law terms in (2) is characterized by a ratio $R$ of the exponential to power law terms integrated over $P_{t}^{2}$ :

$$
R=\frac{A_{e}\left(2 m+2 T_{e}\right)(n-1)}{18.11 A \cdot n \cdot T_{e}}
$$

The ratio $R$ for the inclusive charged particle spectra for $p \bar{p}$ and $p p$ collisions as function of $\sqrt{s}$ is shown in Figure 2a. Interestingly, this ratio is almost independent of the collision energy and equals to about 4 . In Figure $2 \mathrm{~b}$ the ratio $R$ is shown for $A u-A u$ interactions at RHIC as function of centrality of the heavy ions collision. As seen on Figure $2 \mathrm{~b}$ the relative contribution of the exponent term reaches minimum values at medium centralities of heavy ion collisions.

On the other hand, the QCD partonic interaction must describe any hard scattering process like the heavy quark production, or high $Q^{2}$ DIS. Indeed, the spectrum of heavy $J / \Psi$ quarkonium produced in high energy $p \bar{p}$ collisions at Tevatron [17] follow the pure power law distribution and has no room for an exponential term of the parameterization (2). In addition, in the high energy DIS, photoroduction and $\gamma \gamma$ collisions the power law term of the new proposed parameterization (2) dominates the produced particle spectra. Thus, only the inclusive spectra of charged particles produced in pure baryonic collisions require a substantial contribution of the Boltzman-like exponential term.

Finally, a map of the parameters $T$ and $n$ for proton-(anti)proton, heavy ion, gamma-proton and gamma-gamma collision at different energies is displayed in Figure 3.

There are two clearly distinct trends seen in Figure 3. The $p p$ and $p \bar{p}$ collision data show an increase of the $T$-parameter and decrease of the $n$-parameter with collision energy $\sqrt{s}$ increasing. The second trend, where the values of both parameters the $T$ and $n$ increase, is defined mainly by the RHIC $A u-A u$ collision data at $\sqrt{s}=200 \mathrm{GeV}$ per nucleon. In this case a simultaneous increase of the $T$ and $n$ values corresponds to an increase of the centrality (or charged multiplicity) of heavy ion collisions. Surprisingly, the both trends cross each other at medium centralities corresponding to the minimum bias $A u-A u$ collisions and $p \bar{p}$ interactions with energy of $\sqrt{s}=200 \mathrm{GeV}$. Naively one could expect the single $p \bar{p}$ interaction has more similarity to the very peripheral single nucleon-nucleon interactions. Contrary to that naive expectation, the Deep Inelastic Scattering (DIS), $\gamma p$ and $\gamma \gamma$ interaction data with $\sqrt{s}$ ranging approximately from $100 \mathrm{GeV}$ to $200 \mathrm{GeV}$ belong to the second trend shown in Figure 3 and are located on the parameter map (Figure 3) nearby very peripheral heavy ion interactions at about the same collision energy per nucleon. 


\section{Conclusion}

In conclusion we have proposed a new parameterization of the spectrum shape of inclusive charged particles produced in high energy collisions. This new parameterization describes the available experimental data significantly better than the broadly used Tsallis-type parameterization. The proposed parameterization is a sum of an exponential (Boltzman-like) and a power law (Tsallis-like) terms. The parameters of these two terms turned out to be strongly correlated. We observe, that the shapes of the power law terms in minimum bias heavy ion collisions and in proton-(anti)proton interactions at the same collision energy are practically the same. Additionally, the shapes of the power law terms in the spectra measured with very peripheral heavy ion collisions and interactions of high energy particles with photons show very close similarity. The difference in size of the exponential Boltzman-like contribution to these spectra is mainly responsible for the difference in the spectra shapes observed in these experiments.

Due to the space limitations we leaved out several illustrative plots here. The more extended version of this report could be found in [19].

\section{Acknowledgments}

This work was partially supported by Russian Foundation for Basic Research and the Grant of Helmholtz Association HRJRG-02.

\section{References}

[1] B. Alper et al (British-Scandinavian Collaboration), Nucl.Phys. B100, 237, 1975.

[2] C. Albajar et al. (UA1 Collaboration), Nucl.Phys. B335, 261, 1990.

[3] M. Banner, et al. (UA2 Collaboration and Bern-CERN-Copenhagen-Orsay-Pavia-Saclay Collaboration), Z.Phys. C27, 329, 1985.

[4] G. Bocquet et al. (UA1 Collaboration), Phys.Lett. B366, 434, 1996

[5] F. Abe et al. (CDF Collaboration), Phys.Rev.Lett. 61, 1819, 1988

[6] T. Aaltonen et al. (CDF Collaboration), Phys.Rev. D79, 112005, 2009

[7] I. Abt et al. (H1 Collaboration), Phys.Lett. B328, 176, 1994

[8] M. Derrick et al. (ZEUS Collaboration), Z.Phys. C67, 227, 1995.

[9] (L3 Collaboration) Phys. Lett. B 554, 105, 2003

[10] C. Adloff, et al. (H1 Collaboration), Eur.Phys.J, C10, 363, 1999

[11] (OPAL collaboration), Phys.Lett. B651, 92, 2007

[12] C. Adloff, et al. (H1 Collaboration), Nucl.Phys. B485, 3, 1997.

[13] M. Derrick et al. (ZEUS Collaboration), Z.Phys. C70, 1, 1996.

[14] S. S. Adler et al. (PHENIX Collaboration), Phys.Rev. C69, 034909, 2004

[15] V. Khachatryan et al. (CMS Collaboration), JHEP 02, 041, 2010

[16] C.Tsallis, J.Stat.Phys., 52, 479, 1988.

C.Tsallis, Brazilian J. of Rhys., 29, 1, 1999.

[17] D.E. Acosta et al. (CDF Collaboration), Phys.Rev. D71, 032001, 2005.

[18] G.D. Holman et al., ApJ. 595, 97, 2003.

[19] A. Bylinkin, A. Rostovtsev, e-Print: arXiv:1008.0332 [hep-ph]. 

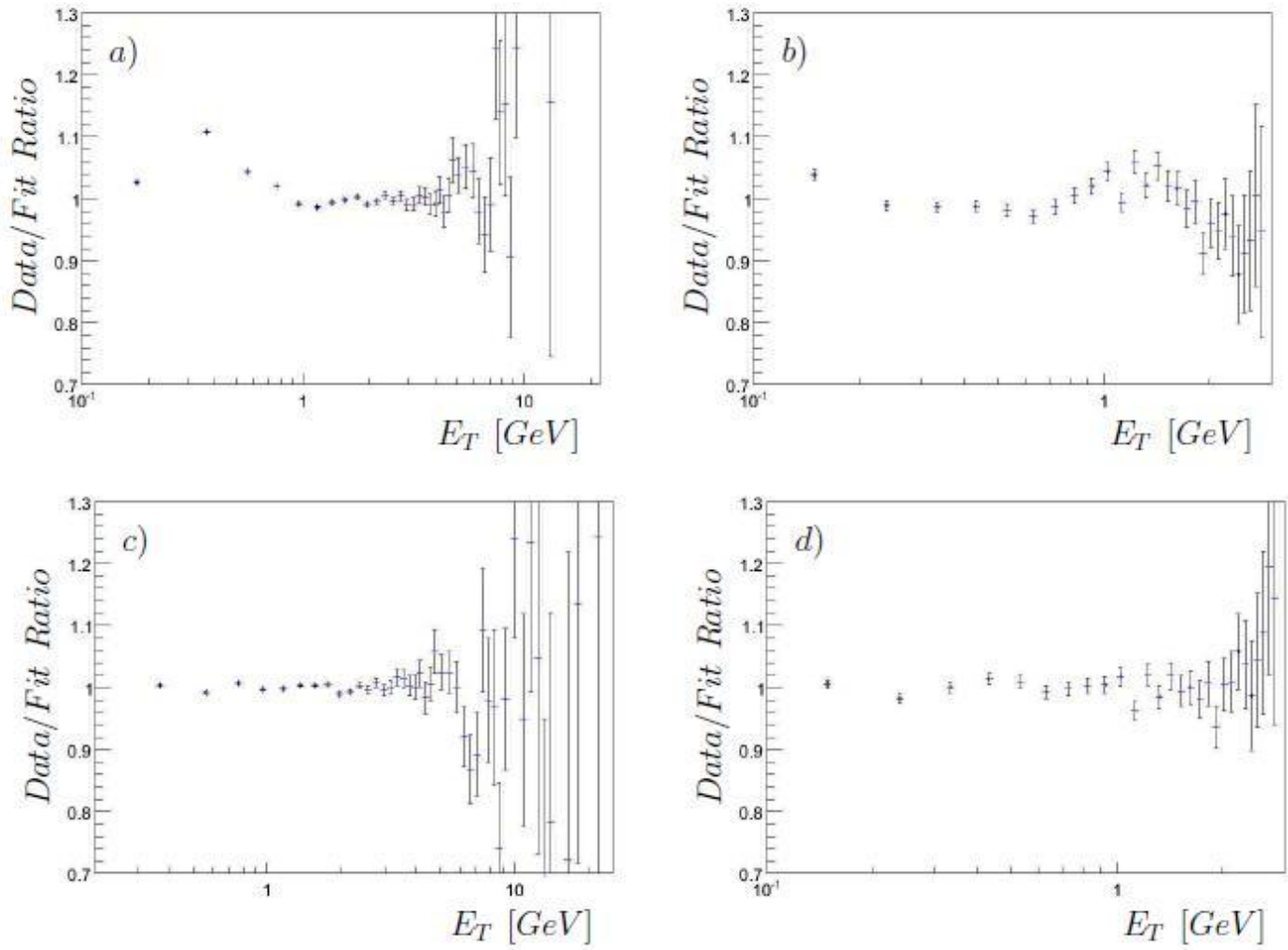

Figure 1: A ratio of the data to the Tsallis-type (1)(a,b) and the modified (2)(c,d) fit functions in $p \bar{p}$ collisions at $\sqrt{s}=630 \mathrm{GeV}$ [4] (a,c) and RHIC $A u-A u$ collisions at $\sqrt{s}=200 \mathrm{GeV} / N[14]$ $(\mathrm{b}, \mathrm{d})$.
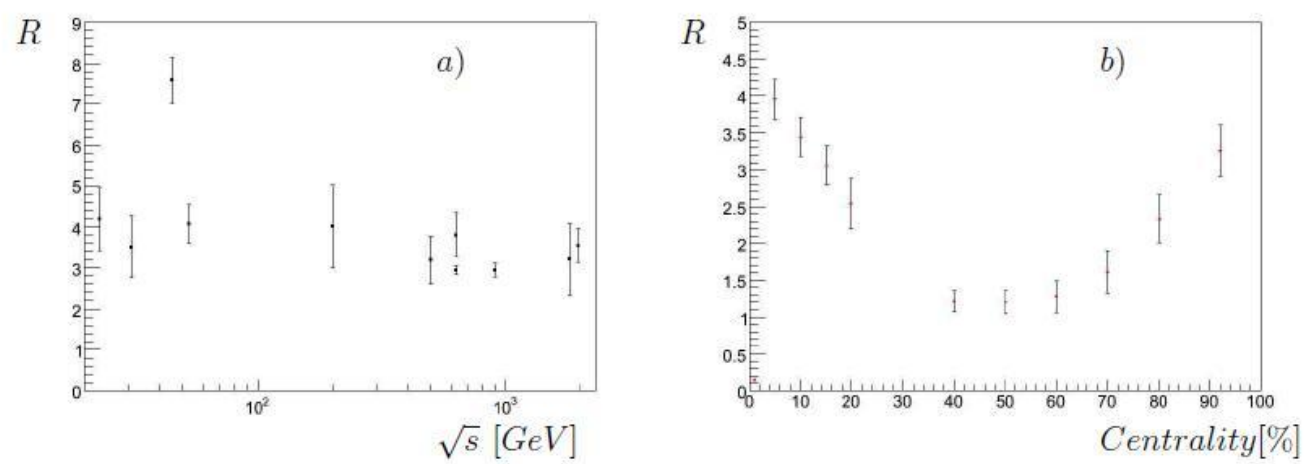

Figure 2: The ratio $R$ of the exponential to power law contributions to the parameterization (2)for $p \bar{p}$ and $p p$ collisions as function of $\sqrt{s}$ (a) and for $A u-A u$ interactions at RHIC as function of centrality (b). 


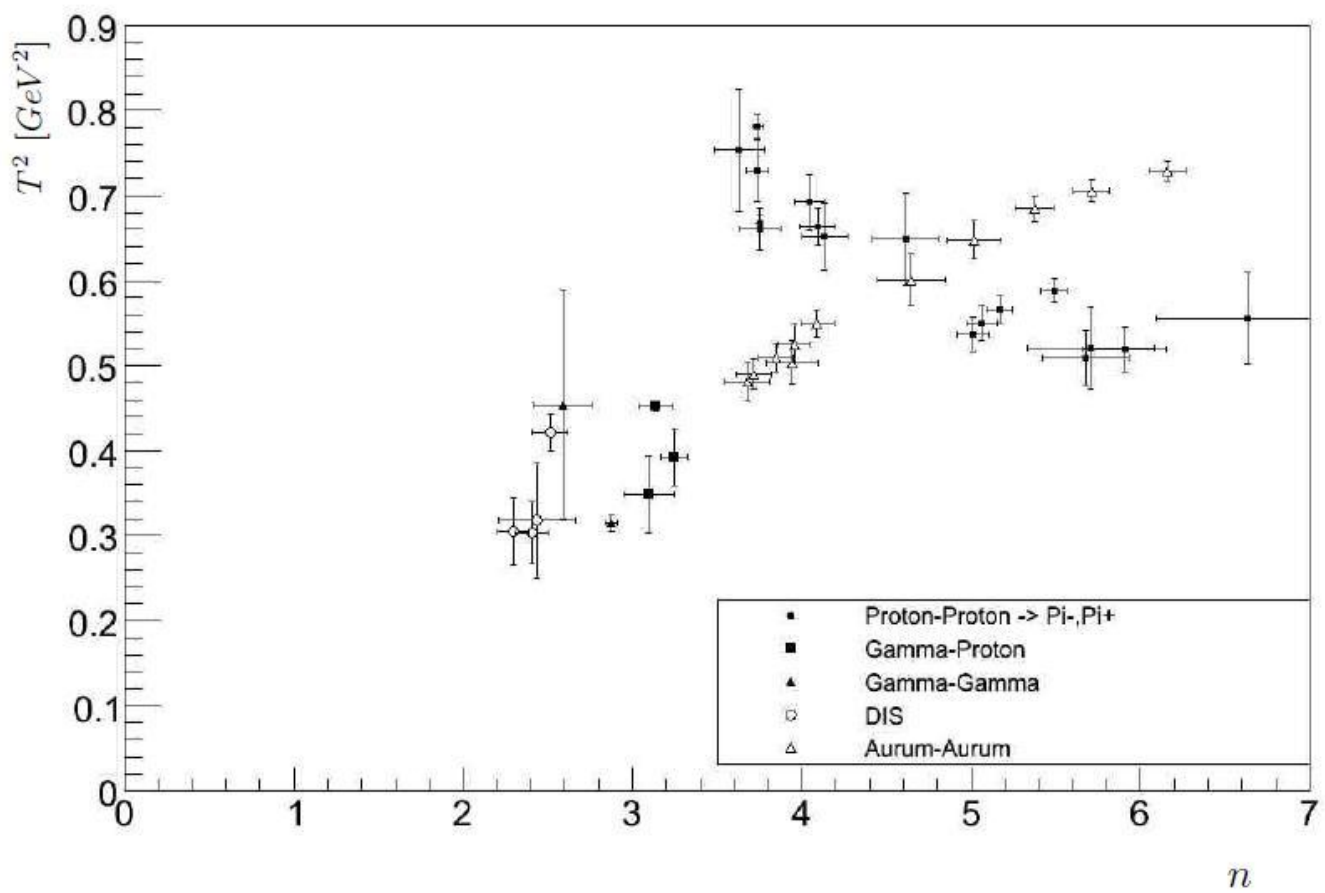

Figure 3: Map of the parameters $T$ and $n$ for proton-(anti)proton, heavy ion, gamma-proton and gamma-gamma collision at different energies. 